\title{
BRAZILIAN TEST OF CONCRETE EVALUATED BY AE
}

\author{
H.W. Reinhardt, F. Finck, C. Grosse and J. Kurz \\ University of Stuttgart, Institute of Construction Materials, Pfaffenwaldring, 70569 Stuttgart, Germany
}

\section{ABSTRACT}

For a detailed evaluation of fracture processes due to tensile stresses using acoustic emission (AE) technique, a splitting test (Brazilian test) is particularly well-suited Thus, a Brazilian test was performed to generate a well-defined crack surface in a concrete cube. Using AE technique, the formation of micro cracks was observed and correlated with the stress distribution during the test. During the experiment, some 1800 acoustic emissions were recorded. Half of these events accompanied the evolution of the main crack. From these 900 events, 378 AE events were localized with an accuracy better than $5 \mathrm{~mm}$.

The well-defined crack in the center of the specimen allowed a mapping of the crack surface. For this purpose, the specimen was ground in a stepwise manner normal to the crack subsequent to testing. The run of the crack in the ground surfaces was digitized from photographs and interpolated. Inversion of AE amplitudes of the spatial displacement distribution yields a system of equivalent moments for selected $\mathrm{AE}$ events, the so-called moment tensors. Analysis of the moment tensor allows an interpretation of failure based on fracture mechanics and gives information about the stress field within the specimen.

\section{INTRODUCTION}

For a detailed evaluation of fracture processes due to tensile stresses using acoustic emission technique, a splitting test (Brazilian test) is particularly well-suited(Finck et al. [1]). During the experiment presented in this article some 1800 acoustic emissions were recorded. Half of these events accompanied the evolution of the main crack. From these 900 events 378 were localized with an accuracy better than $5 \mathrm{~mm}$. The well-defined crack in the center of the specimen allowed a mapping of the crack surface. For this purpose, the specimen was ground in a stepwise manner normal to the crack subsequent to testing. The run of the crack in the ground surfaces was digitized from photographs and interpolated. An advanced 3D visualization routine combines the results of the different analysis methods.

\section{EXPERIMENTAL SETUP}

To generate controlled tensile failure, a splitting test (DIN 1048 [2]) with a concrete cube (edge length $200 \mathrm{~mm}$ ) was performed. Compressive load was applied using two parallel steel edges, one on the top and the other on the bottom of the specimen. This produced a tensile crack parallel to the steel edges. A controlled opening of the tensile crack is necessary to obtain as many acoustic emissions during the evolution of the crack as possible. Therefore, the crack opening was measured by two linear displacement sensors (LVDT), one on each side of the cube. The mean crack opening acted as the control parameter for the loading device.

Acoustic emissions were registered by eight piezo-electric ultrasonic sensors and recorded by a transient recorder with a sample frequency of $5 \mathrm{MHz}$ per channel and an amplitude resolution of 12 Bit. Additionally, piston displacement, load and crack opening were recorded.

According to DIN EN 206, the concrete had a strength grade of C35/45. The maximum aggregate size was $16 \mathrm{~mm}$. Before the test, a compressive wave (P-wave) velocity of $4600 \mathrm{~m} / \mathrm{s}$ was determined for the specimen. 


\section{EVALUATION OF RESULTS}

The analysis of the data obtained during the splitting test can be divided into four categories:

(1) Analysis of mechanical data and the acoustic emission rate,

(2) localization of acoustic emissions,

(3) evaluation of the topography of the fracture plane,

(4) fracture mechanical studies on moment tensors.

In the following sections, results of the experiment are presented and discussed.

\subsection{Mechanical Test Data}
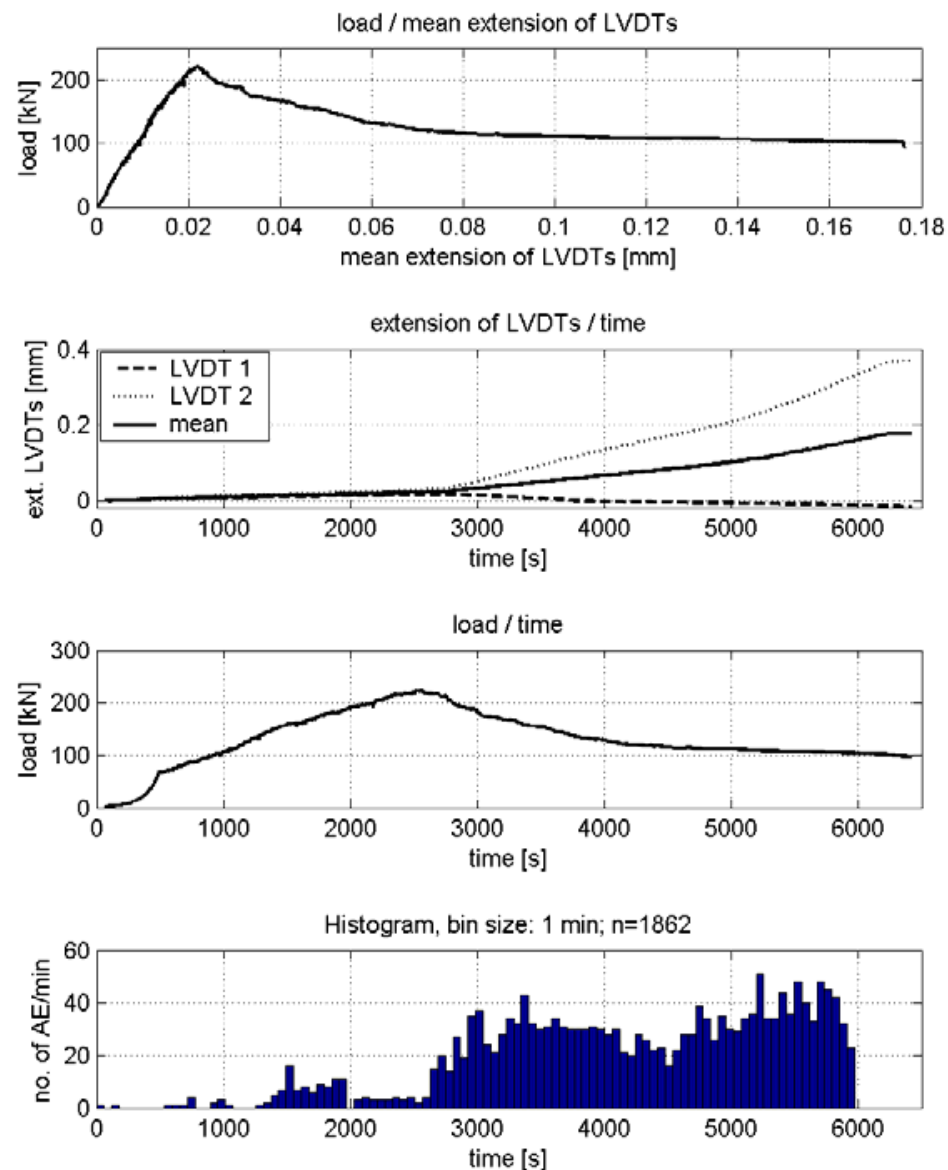

Figure 1: Mechanical test data and a histogram of the acoustic emission activity during the test

In figure 1 the mechanical test data and the acoustic emission rate over the test period are illustrated. The first plot contains the load as a function of the mean crack opening. It can be seen that the first phase shows a more or less linear elastic behavior until the load decreases with an 
increasing crack opening. Towards the end of the test the crack opens with a behavior similar to creep at a nearly constant load.

The second and third plots show the load over time and displacement over time behavior, respectively. LVDT 2 was attached on the backside of the specimen, LVDT 1 on the front. The crack opening started from the back of the specimen and grew through the cube to the front side. With an advanced opening of the crack, LVDT 1 showed negative values. The negative values occurred because the deformation of the specimen led to a compression of LVDT 1. No crack was visible on the front side of the specimen until the end of the experiment. In the load versus time curve, the compression of the felt strip between the specimen and the steel edges at the application of load can be seen during the first 500 seconds. After that time, load increased nearly linearly, with a small break at about 1500 seconds, which is also accompanied by acoustic emissions (see histogram).

The histogram on the bottom of figure 1 gives the acoustic emission rate per minute. The trigger times of all registered acoustic emissions are taken into account. Because the rate of events was well-below the capacity of the acquisition system, it can be assumed that no data was missed. In addition to the mentioned activity reflected in the minor break in the ascending branch of the load versus time curve, the acoustic emission rate increases after the achievement of maximum load. From that point the crack grows through the specimen, accompanied by numerous acoustic emissions. The splitting tensile strength amounted to $3.52 \mathrm{MPa}$.

\subsection{Localization}

Acoustic emissions are observed by a number of sensors with well-defined positions and the Pwave velocity has to be measured. For the estimation of the focal coordinates of an acoustic emission the accurate onset times of the P-waves must be determined. A simple system of linear equations is the basis for this analysis. Since the system of equations is overdetermined when data from eight sensors is available, the run time residuals are minimized with the method of least squares. This leads to improved accuracy and yields error bounds for the estimated focal coordinates (Grosse et al. [3]). From the 1800 acoustic emissions registered, the onset times of the first 900 events were picked with the WinPecker ${ }^{\odot}$ software (Grosse, [4]). From these events, 378 could be localized with an accuracy better than $5 \mathrm{~mm}$.
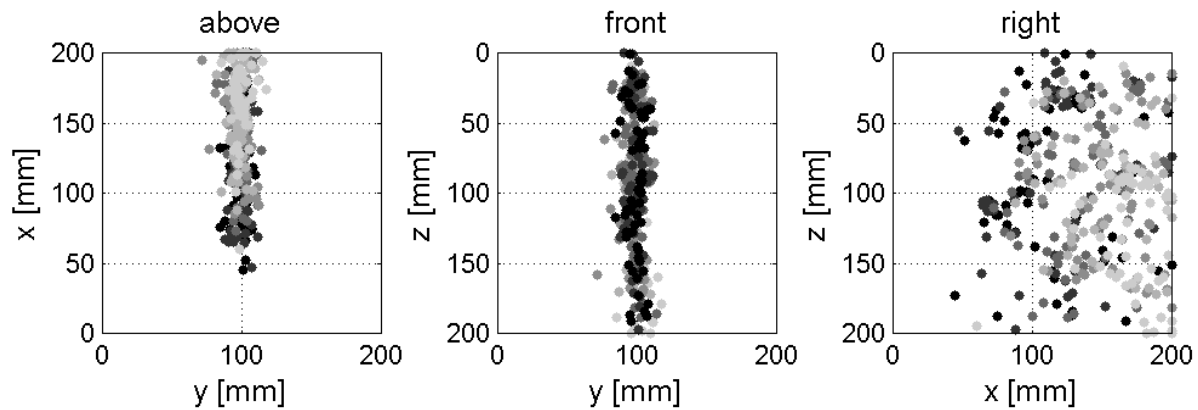

Figure 2: Projections of the localization of acoustic emissions onto the $x / y, y / z$ and $x / z$ planes. Events marked in light grey occurred at the beginning of the test, dark ones at an advanced stage of loading 
In figure 2, results of the localization are illustrated. The events are projected onto the main coordinate planes revealing a view from above, from the front and the right side of the specimen. The brightness of the data points provides information about the time when the events occurred. Light grey represents events from the beginning of the test, dark grey represents events from an advanced stage. The last localized event occurred at 4246 seconds after the start of loading.

The acoustic emissions are located along a well-defined zone of failure parallel to the steel edges applying compressive load. Local damage at the point of load application was minimized through the use of felt strips which evenly spread the applied compressive load. Consequently, only a small number of acoustic emissions were visible in the region where load was applied. It is possible, however, that some of the acoustic emissions from this regions were ignored by the localization process due to a weak signal to noise ratio.

Considering the brightness of the data points, the growth of the crack from the back to the front is clearly visible. Even at an advanced stage of the test, where the crack front had progressed through the specimen, acoustic emissions occurred at the back side of the specimen, where the crack was open already. This can be explained by either an increasing accretion of micro cracks or a rubbing of the crack surfaces.

\subsection{Topography of the Fracture Plane}

The splitting test performed revealed a well-localized and visible crack in the middle of the specimen. For a correlation of this macroscopic crack with the localization of the acoustic emissions (i.e. micro cracks) along that zone of failure, the topography of the crack surface was investigated. The specimen was ground from the top in steps of $5 \mathrm{~mm}$. The surfaces of the various steps were photographed. From the digitized run of the crack in every section, a fracture plane was interpolated. The digitization process of the crack has an error of approximately $2 \mathrm{~mm}$.

In most of the sections only a single crack was visible. The crack went through the concrete matrix from one aggregate to the next. Depending on the strength of the grains, they were divided or the crack ran around them, which caused the crack surface to undulate.

The localization of the acoustic emissions revealed, that micro cracking and debonding also occurred in a distance of several millimeters to the final macro crack.

\subsection{Fracture Mechanics}

The basics of evaluating the moment tensor have been presented in the references (Andersen [5], Finck [6], Grosse [7], Jost and Hermann [8]). Two clusters containing 5 and 9 events, respectively, were selected from the localized events. Since the Green's functions, representing the transfer functions of the medium are eliminated in the relative moment tensor inversion, the radii of these clusters have to be small enough and the events should originate in a relatively short temporal interval. Low frequency noise from the data was minimized by a suitable wavelet filter algorithm. The transient raw data, representing velocity over time, were then integrated to reveal the displacement. The first half-wave amplitudes of the longitudinal P-waves were picked from the data for input.

An overview of the results of the moment tensor inversion is presented in the form of the radiation patterns in stereographic projections (figure 3). All events in cluster C2 show a similar behavior, with tension (dark) more or less parallel to the y-axis. A small compressive component almost parallel to the $\mathrm{z}$-axis is indicated by negative amplitudes (light colored). 


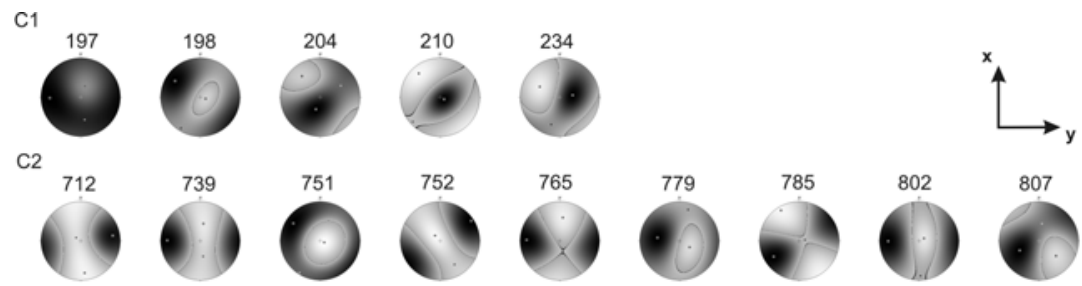

Figure 3: Radiation patterns of all moment tensors from clusters C1 and C2

The decomposition of the moment tensors is revealing the percentile contributions of isotropic, double couple and CLVD mechanisms. Significant positive isotropic components and a mixture of double couple and CLVD mechanisms are evident. The solutions for cluster C1 are not that consistent. The directions of positive amplitudes due to tensile stress vary more around the $\mathrm{y}$ axis than in C2. The decomposition shows similar results as in cluster C1. Event 210 has a negative isotropic component, which could be explained by a pore collapse in combination with shear.

The results corroborate the assumption of mode I failure with a major tensile crack parallel to the steel edges from which load was applied. The mean tensile stress axis is parallel to the yaxis, which is also the preferred direction of positive amplitudes.

\section{SUMMARY}

A splitting test was performed to generate controlled failure in a concrete specimen. During the test, acoustic emissions were recorded for a detailed investigation of the damage zone. The configuration of the stress field produced a well-defined crack, which allowed for the mapping and digitization of the topography of the crack surface. The macro crack ran from the back to the front side of the specimen. This was observed in the mechanical data, as well as in the results of the localization.

For an evaluation of the stress field and the fracture mechanisms leading to failure, moment tensors were calculated. In the moment tensors a significant positive isotropic component was observed and the deviatoric component consisted primarily of a double couple. The direction of maximum tension in the radiation patterns was almost parallel to the mean tensile stress generated by the experimental setup. A small compressive component was found parallel to the z-axis.

The investigations reveal tensile fracturing as the dominant cause of failure. However, variations of the tension axes and deviations from pure tensile failure are observed. Inhomogeneities, such as aggregates, generate undulations of the crack surface and can be responsible for an enhanced complexity of micro cracking. Corresponding shear stresses would explain the significant double couple components.

The projection of the seismic radiation patterns onto focal spheres in a 3-dimensional image depicts the results of the moment tensor inversion. These projections can be combined with the results of the localization and other investigations and allows for a comprehensive understanding of failure mechanisms in a specimen.

Signal-based analysis of acoustic emissions is of course more time consuming than the classic parameter-based approach, where a range of parameters is extracted automatically and a rough localization is sometimes possible. However, signal-based analysis allows for much more detailed evaluation of failure processes. Thus, the accuracy of the localization is enhanced. Furthermore, the localization can be automated for a number of events using suitable de-noising 
algorithms and automatic onset pickers. Important knowledge about the stress field and fracture mechanics can be obtained from the data. A broad understanding of the radiation pattern of seismic energy and an automation of the data processing is the basis for future applications in structural health monitoring.

\section{ACKNOWLEDGEMENT}

This work was funded by the Deutsche Forschungsgemeinschaft DFG (SFB 381, TP A6).

\section{REFERENCES}

[1] Finck, F, Yamanouchi, M., Reinhardt, H.-W. and Grosse, C.U.: Evaluation of mode I failure of concrete in a splitting test using acoustic emission technique. Int. Journal of Fracture, 2004 (in print).

[2] DIN 1048, part 5: Prüfverfahren für Beton - Festbeton, gesondert hergestellte Probekörper. Deutsches Institut für Normung e. V, 1991.

[3] Grosse, C.U., Reinhardt, H.-W. and Finck, F.: Signal-Based Acoustic Emission Techniques in Civil Engineering. Journal of Materials in Civil Engineering (ASCE) 15 (3), pp. 274 - 279, 2003.

[4] Grosse, C.U.: WinPecker - Programm zur vollautomatischen dreidimensionalen Lokalisierung von Schallemissionsquellen. In: 12. Kolloquium Schallemission, Jena. DGZfP Berichtsband 72, pp. $191-204,2000$.

[5] Andersen, L.M.: A relative moment tensor inversion technique applied to seismicity induced by mining. Ph.D. thesis, University of the Witwatersrand, Johannesburg, South Africa, 2001.

[6] Finck, F.: Application of the moment tensor inversion in material testing. Otto-Graf-Journal 12, pp. 145 - 156, 2001.

[7] Grosse, C.U.: Grundlagen der Inversion des Momententensors zur Analyse von Schallemissionsquellen. In: Werkstoffe und Werkstoffprüfung im Bauwesen. Festschrift zum 60. Geburtstag von Prof. Dr.-Ing. H.-W. Reinhardt. Libri BOD, Hamburg, pp. 82 - 105, 1999.

[8] Jost, M.L. and Hermann, R.B.:, A students guide to and review of moment tensors. Seism. Res. Letters 60, pp. 37 - 57, 1989 